\title{
Correction to: Perfect Narrowband Absorber Based on Patterned Graphene-Silica Multilayer Hyperbolic Metamaterials
}

\author{
Yan Feng ${ }^{1} \cdot$ Zheng-Da Hu$^{1}$ • Aliaksei Balmakou ${ }^{2}$ - Sergei Khakhomov ${ }^{2} \cdot$ Igor Semchenko ${ }^{2} \cdot$ Jicheng Wang $^{1,3}$. \\ Dongdong Liu ${ }^{4} \cdot$ Tian Sang ${ }^{1}$
}

Published online: 2 July 2020

(C) Springer Science+Business Media, LLC, part of Springer Nature 2020

\section{Correction to: Plasmonics}

$$
\text { https://doi.org/10.1007/s11468-020-01202-x }
$$

The original version of this article unfortunately contained a mistake. The image of Fig. 7 is wrong and the same as Fig. 8. The corrected figure is given below. The original article has been corrected.

Publisher's Note Springer Nature remains neutral with regard to jurisdictional claims in published maps and institutional affiliations.

The online version of the original article can be found at https:/doi.org/ $10.1007 / \mathrm{s} 11468-020-01202-\mathrm{x}$

Jicheng Wang

jcwang@jiangnan.edu.cn

$\triangle$ Tian Sang

sangt@jiangnan.edu.cn

1 School of Science, Jiangsu Provincial Research Center of Light Industrial Optoelectronic Engineering and Technology, Jiangnan University, Wuxi 214122, China

2 Department of Optics and General Physics, Francisk Skorina Gomel State University, Sovetskaya Str. 104, 246019 Gomel, Belarus

3 National Laboratory of Solid State Microstructures, Nanjing University, Nanjing 210093, China

4 School of Mathematics \& Physics Science, Xuzhou University of Technology, Xuzhou 221018, China 


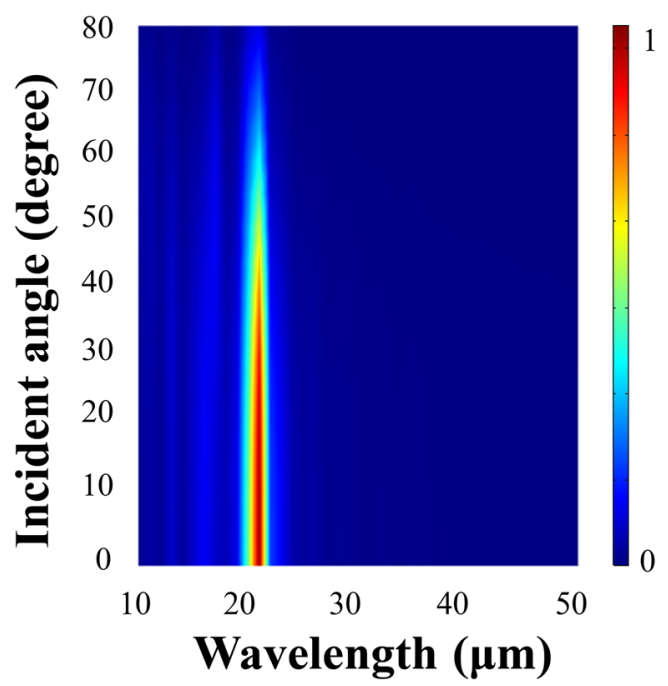

Fig. 7 Absorptance of the absorber vs wavelength when varying the angle of incident light $\theta$ 